\title{
Cholinergic stimulation may mitigate arrhythmic risk during treatment of COVID-19
}

\section{Editorial}

The COVID-19 pandemic led scientists all over the world to try finding potential pharmacologic options to treat infected patients. In this context, old antimalarial drugs as chloroquine and hydroxychloroquine has emerged as promising treatments.

Physicians became heroes during the pandemic, and heroes feelimpotent when only supportive measurements are available. Heroes fight, blindly and hopefully prescribing drugs not proven to be effective for treating COVID-19. While scientists claimed that there was not enough scientific evidence supporting the use of these medicines in patients infected by the SARS-Cov-2, politicians and lay people argument that even without solid evidences for the benefits, there would be nothing to lose if these drugs were prescribed.

Chloroquine and hydroxychloroquine are known to prolong QTc interval and cause torsades de pointes polymorphic ventricular tachyarrhythmia. This happens through the blockage of the humanether-a-go-go-related gene (hERG) which consequently blocks the rapidly activating delayed rectifier $\mathrm{K}^{+}$current. $^{1}$

In a series of 95 patients $^{2}$ suspected of having COVID-19, with normal QTc interval, and who received chloroquine, there was a mean QTc prolongation of $35 \mathrm{~ms}$. Although none of these patients presented ventricular arrhythmias, $23 \%$ of them presented a QTc interval exceeding $500 \mathrm{~ms}$.

Compassionate use of drugs may be an alternative in the "no man's land" where we all live in now. The United States Food and Drug Administration (FDA) have authorized the use of chloroquine and hydroxychloroquine in hospitalized patients through an Emergency Use Authorization during the COVID-19 pandemic, where clinical trials with these drugs are not available. ${ }^{3}$

Adverse effects to these unproven treatments should, if possible, be prevented or at least closely monitored.The Canadian Heart Rhythm Society has recently published guidelines ${ }^{4}$ on minimizing the risk of drug-induced ventricular arrhythmia during treatment of COVID-19. The proposed mitigation focus on identifying patients at risk for developing QTc interval prolongation secondary to chloroquine or hydroxychloroquine, prevent electrolyte disturbances and not use other drugs also known to prolong QTc concomitantly.

The recommendation for drug induced QTc prolongation is drug discontinuation. If chloroquine or hydroxychloroquine prove to be effective against COVID-19, risk and benefit would have to be individually assessed, unless there are drugs that can be prescribed and reduce the risk of malignant arrhythmia.

Beta-blockers are recommended in patients with long QT syndrome (LQTS) with a resting QTc greater than 470 ms. ${ }^{5}$ A metaanalysis showed that beta-blockers were effective in reducing risk of cardiac events in patients with LQTS, ${ }^{6}$ but different beta-blockers showed different effectiveness in preventing arrhythmias. Whereas nadolol reduced risk in patients with LQTS1 and LQTS2, atenolol and propranolol were only effective in LQTS1 and metoprolol showed no reduction in arrhythmias. ${ }^{6,7}$
Volume 5 Issue 4 - 2020

\author{
Renata RT Castro, ${ }^{1,2,3}$ Marco Antonio Orsini \\ Neves,' João Giffoni da Silveira Neto, ${ }^{1,3}$ \\ Roberta RT Castro' \\ 'School of Medicine, Universidade Iguaçu, Brazil \\ ${ }^{2}$ Cardiology Clinic, Hospital Naval Marcílio Dias, Brazilian Navy, \\ Brazil \\ ${ }^{3}$ Cardiologia do Esporte, Brazil
}

Correspondence: Renata RT de Castro, School of Medicine, Universidade Iguaçu, Cardiology Clinic, Hospital Naval Marcílio Dias, Cardiologia do Esporte, Brazil, Email castrorrt@gmail.com

Received: July 15, 2020 | Published: July 202020

Drugs with cholinomimetic effect are expected to reduce heart rate and conduction times, ${ }^{8}$ showing potential antiarrhythmic benefits. Pyridostigmine is a reversible anticholinesterase agent used for the treatment of myasthenia gravis. When used in health adults pyridostigmine reduces rest QTc dispersion ${ }^{9}$ and lowers heart rate during rest and exercise without hypotension..$^{9,10}$ In patients with coronary artery disease pyridostigmine reduces QTc interval ${ }^{11}$ and delays the onset of exercise-induced myocardial ischemia, inhibiting chronotropic response to exercise and improving peak exercise tolerance. ${ }^{12}$ It also reduces QTc interval during recovery from maximal exercise in ischemic heart disease. ${ }^{13}$ Zimerman et al. ${ }^{14}$ conducted an elegant protocol where cardiac electrophysiologic studies were conducted before and 90-120 minutes after the oral administration of pyridostigmine and concluded that the drug increased the ventricular refractory period at higher heart rates without provoking conduction disturbances. Finally, pyridostigmine reduced the incidence of nonsustained ventricular arrhythmias in patients with Chagas cardiac disease..$^{15}$

These results show promising effectiveness of pyridostigmine in reducing the risk of drug induced QTc prolongation. Whether pyridostigmine can be used with chloroquine or hydroxychloroquine to counteract QTc prolongation remains to be proved in further studies.

\section{Acknowledgements}

None.

\section{Conflicts of interest}

The authors declare no conflicts of interest.

\section{References}

1. Traebert M, Dumotier B, Meister L, et al. Inhibition of hERG K+ currents by antimalarial drugs in stably transfected HEK293 cells. Eur J Pharmacol. 2004;484:41-48.

2. van den Broek MPH, Möhlmann JE, Abeln BGS, et al. Chloroquineinduced QTc prolongation in COVID-19 patients. Neth Heart J. 2020:14. 
3. FDA. FDA cautions against use of hydroxychloroquine or chloroquine for COVID-19 outside of the hospital setting or a clinical trial due to risk of heart rhythm problems. FDA; 2020.

4. Sapp JL, Alqarawi W, MacIntyre CJ, et al. Guidance on minimizing risk of drug-induced ventricular arrhythmia during treatment of covid-19: statement from the canadian heart rhythm society. Can J Cardiol. 2020.

5. Al-Khatib SM, Stevenson WG, Ackerman MJ, et al. 2017 AHA/ACC/ HRS guideline for management of patients with ventricular arrhythmias and the prevention of sudden cardiac death: executive summary: a report of the american college of cardiology/american heart association task force on clinical practice guidelines and the heart rhythm society. $J \mathrm{Am}$ Coll Cardiol. 2018;72:1677-1749.

6. Ahn J, Kim HJ, Choi JI, et al. Effectiveness of beta-blockers depending on the genotype of congenital long-QT syndrome: A meta-analysis. PLoS One. 2017;12:e0185680.

7. Abu-Zeitone A, Peterson DR, Polonsky B, et al. Efficacy of different beta-blockers in the treatment of long QT syndrome. J Am Coll Cardiol. 2014;64:1352-1358.

8. Prystowsky EN, Jackman WM, Rinkenberger RL, et al. Effect of autonomic blockade on ventricular refractoriness and atrioventricular nodal conduction in humans. Evidence supporting a direct cholinergic action on ventricular muscle refractoriness. Circ Res. 1981;49:511-518.
9. Castro RR, Serra SM, Nobrega AC. Reduction of QTc interval dispersion. Potential mechanism of cardiac protection of pyridostigmine bromide. Arq Bras Cardiol. 2000;75:205-213.

10. Serra SM, Costa RV, Bastos BG, et al. Exercise stress testing in healthy subjects during cholinergic stimulation after a single dose of pyridostigmine. Arq Bras Cardiol. 2001;76:279-284.

11. Castro RR, Porphirio G, Serra SM, Nobrega AC. Cholinergic stimulation with pyridostigmine reduces the QTc interval in coronary artery disease. Braz J Med Biol Res. 2002;35:685-689.

12. Castro RR, Porphirio G, Serra SM, et al. Cholinergic stimulation with pyridostigmine protects against exercise induced myocardial ischaemia. Heart. 2004;90(10):1119-1123.

13. Castro RR, Serra SM, Porphirio G, et al. Pyridostigmine reduces QTc interval during recovery from maximal exercise in ischemic heart disease. Int J Cardiol. 2006;107:138-139.

14. Zimerman LI, Liberman A, Castro RR, et al. Acute electrophysiologic consequences of pyridostigmine inhibition of cholinesterase in humans. Braz J Med Biol Res. 2010;43:211-216.

15. de Castro RRT, Porphirio G, Xavier SS, et al. Cholinesterase inhibition reduces arrhythmias in asymptomatic Chagas disease. Cardiovasc Ther. 2017. 